\title{
Effects of Coenzyme Q10 Administration in Amyotrophic Lateral Sclerosis (ALS). Report of a Case and Review
}

\author{
Terukazu Kawasaki $^{1}$, R.B. Singh*, ${ }^{*}$, Cornelissen Germaine ${ }^{3}$ and Franz Halberg ${ }^{3}$ \\ ${ }^{1}$ Center for Health and Sports Science, Kyushu Sangyo University, Fukuoka, Japan \\ ${ }^{2}$ Halberg Hospital and Research Institute, Moradabad, India \\ ${ }^{3}$ Halberg Chronobiological Center, University of Minnesota Medical School, Minneapolis, USA
}

\begin{abstract}
Objective: Coenzyme Q10 (CoQ10) appears to be a promising agent in neurodegenerative disorders because they are associated with defects in the inner mitochondrial membrane and in oxidative phosphorylation. This report aims to highlight the role of CoQ10 in amyotrophic lateral sclerosis.

Methods: A 75 year old medical scientist $\left(1^{\text {st }}\right.$ author TK) unbiased for treatment with CoQ10, volunteered for CoQ10 administration. He himself recorded his symptoms, mood, sensations, body weight, using limb Norris score, hand grip power for both hands. He received highly bioavailable CoQ10 in a dose of $500 \mathrm{mg}$ twice daily initially followed by 200mg twice daily for treatment in 2005. (solubilized ubiquinol, Tishcon Corp. NY). His plasma CoQ10 level was obtained initially and after treatment with CoQ10.

Results: He presented with weakness of left leg along with cramps in the left calf muscle with steppage gate in September 2000. He had wasting of muscles, fasciculations and exaggerated reflexes. He had rapid deterioration in hand grip power in the left hand and weakness in left lower limb followed by right limbs with inability in walking and routine activities. Treatment with ubiquinol in 2005, resulted in better hand grip power, mood and sensation within a month and in 2006, he reported that hand grip power and wasting of muscles are not progressing as rapidly as before administering CoQ10.
\end{abstract}

Interpretations: Treatment with highly solubilized ubiquinol can provide benefit to patients with ALS. Further controlled trials would be necessary to confirm our findings.

Keywords: Motor Neuron Disease, diet, ubiquinol, wasting of muscles.

\section{INTRODUCTION}

There is significant interest in exploring the use of antioxidants, particularly coenzyme Q10 (CoQ10) for the potential treatment of neurodegenerative disorders [1-7]. CoQ10 and its analog, idebenone as well as antioxidant vitamin tocopherol, monoamine oxidase inhibiters selegiline have been widely used as neuroprotectants in the treatment of neurodegenerative and neuromuscular disorders [6-13]. However, CoQ10 appears to be a most promising neuroprotectant in several neurological degenerative diseases because Alzheimer's disease, Parkinson's disease, and other neurodegenerative disorders were found to have defects in the inner mitochondrial membrane and in oxidative phosphorylation [6-10]. A decrease in CoQ10 levels and in complex I activity have also been reported; leading to the hypothesis that such changes may play a role in the pathogenesis of neuronal damage [4-9]. CoQ10 is an endogenous proenzyme found in the inner mitochondrial

*Address correspondence to this author at the Halberg Hospital and Research Institute, Civil Lines, Moradabad-10(UP)244001, India;

Fax: 0091591 2417437; E-mail: rbs@tsimtsoum.net membrane of human cells [1-4]. It is a fat-soluble quinone that in human tissue has 10 five-carbon isoprenoid units, hence the term CoQ10. Oxidative phosphorylation is an essential process used in the production of energy to sustain organs such as the brain, heart, muscles, and kidneys through the production of adenosine triphosphate (ATP) [6, 7]. CoQ10 is normally involved in a series of enzymatically catalyzed sequential reactions necessary to carry out oxidative phosphorylation via the electron transport chain, which also known as the respiratory chain. It collects reducing electrons from flavoprotein. In a case control study among 1700 subjects, antioxidant vitamin deficiency and oxidative stress have been reported in patients with noncommunicable diseases including degenerative diseases of the brain [2]. Singh and Singh reported beneficial effects by treatment with CoQ10, in a patient with ALS and in a cases with polymyositis and tuberous sclerosis [3]. Since Co Q10 administration increases brain mitochondrial concentrations and exerts neuroprotective effects [6], in this volunteer who is a medical scientist (TK), we report more detailed effects of CoQ10 in ALS. 


\section{CASE REPORT}

A 75 year old medical scientist presented with weakness of left lower limb and cramps in left calf of about one month duration in September 2000. The onset of the disease was insidious and gradually progressive. He complained of wasting of muscles and fasciculations. He had rapid deterioration in hand grip power in the left hand and weakness in left lower limb, followed by right hand and right lower limb with inability in walking and routine activities. Past and family history were not relevant. There was no history of persistent fever or head injury with loss of consciousness.

\section{Physical Examination}

Physical examination revealed pulse 75 per min regular, blood pressure 130/85 mm of $\mathrm{Hg}$ and systemic examination showed no abnormality except in the nervous system. He had weakness, wasting and fasciculations with exaggerated reflexes. The wasting of muscles was more marked in calf and thigh muscles more in left side than right which made him unable to walk normally. There were diminished hand grip and power in the lower limbs and hand more in the left side than in right, which is consistent with the diagnosis of ALS. There was no sensory deficit and power was approximately grade III-IV/V in all the 4 limbs and 0-II in thumbs and little fingers but he was able to type and send emails without much difficulty. Higher centers and sensory centers were within normal limits and he is able to communicate normally on telephones. The diagnosis of myopathy was excluded by a negative electromyogram indicating chronic denervation and muscle biopsy showing no evidence of myopathy? Nerve conduction velocity was within normal limits.

\section{Laboratory Data}

Blood examination conducted in Sept 2000 showed no abnormality in hemoglobin, total and differential leukocyte count, ESR, serum creatinine, C-reactive protein, creatine phosphokinase, blood urea nitrogen and fasting blood glucose. Urine examination for Bence-Jones proteins was negative. Radiological examination of the cervical spine and computerized axial tomography of the brain showed no abnormality. His blood examination for Co Q10 showed increase in CoQ10 concentration after treatment. Cerebrospinal fluid examination showed no abnormality in proteins, sugar and cells.

\section{CLINICAL COURSE}

His clinical course of ALS is given. He was advised Laennec injection (placenta) after diagnosis of ALS at the University hospital but his clinical condition deteriorated rapidly showing marked decrease in body weight, weakness, wasting of muscles and power during routine activities during the next one year. He contacted other coauthors in 2004-2005 because one of us (RBS) had experience in CoQ10 administration in patients with neurodegenerative diseases [3]. After permission from the Institutional ethic committee, he volunteered to take $400-1000 \mathrm{mg} /$ day of solubilized ubiquinol (COQ10) beginning with 200mg twice daily with fatty meals followed by $500 \mathrm{mg}$ twice daily and finally 200mg twice daily after improvement which he is still continuing. Adverse effect of CoQ10 were not observed. However he felt moderate benefit after receiving CoQ10 for 4 weeks which motivated him to take $500 \mathrm{mg}$ twice daily of CoQ10 (total 1000mg/day).

\section{COMMENTS}

This patient who is a medical scientist (TK) presented with weakness, wasting and fasciculations with exaggerated reflexes more in the left limbs than right limbs and diminished hand grip in the left hand, which is consistent with ALS. There was no sensory deficit and power was approximately grade III-IV/V in all the 4 limbs and 0 -II in thumbs and little fingers but he was able to type and send emails without much difficulty. In view of above findings without involvement of higher centers and sensory centers and in absence of myopathy, a clinical diagnosis of ALS was quite relevant.

He was advised Laennec injection (placenta) after the diagnosis of ALS at the University hospital, without any improvement in the next one year. There are only few studies to compare our observations with others. However, a case of ALS was administered CoQ10, $60 \mathrm{mg}$ thrice daily (180mg/day,Q gel, Tishcon Corp,NY) in 1998 by Singh and Singh who showed beneficial effects within one week [3]. There was a symptomatic improvement in weakness and an increase in power in the lower and upper limbs. This patient also had oxidative stress showing higher levels of malondialdehyde, thiobarbituric acid reactive substances and diene conjugates and a deficiency of vitamin C, E and beta carotene which showed improvement after treatment with CoQ10 for 4 weeks [3]. This patient reported improvement in speech, swallowing, walking and general well being. In the present case, these investigations were not done. However, results of CoQ10 administration indicate that solubilized ubiquinol in high doses of $1000 \mathrm{mg} /$ day can be tolerated without any adverse effects. CoQ10 can provide benefits in muscle power and general well being and inhibit further deterioration in muscle wasting and power on long term administration.

In clinical and experimental studies, CoQ10 status has been studied in neurodegenerative diseases and it is administered for treatment with possible beneficial effects or no effect [14-26]. Singh and Singh also reported beneficial effects of CoQ10 in a case with polymyositis [3]. In a dose assessment trial, 31 ALS patients received Co Q10, formulated with $300 \mathrm{IU}$ of vitamin $\mathrm{E}$ on a monthly dosage escalation scale (1200-3000 mg/day). CoQ10 was found to be safe and well-tolerated at the maximum dosage [22]. There were no significant differences between CoQ10 and historical placebo groups in strength, grip, forced vital capacity, or ALS functional rating scale scores compared to placebo group [22]. These pilot data have yielded a followup trial by Kauffman et al with an adaptive, two-stage, biasadjusted, randomized, placebo-controlled, double-blind, Phase II design $(n=185)$ [23]. The primary outcome in both stages was decline in the ALS Functional Rating Scale- 
revised (ALSFRSr) score over 9 months. Stage 1 (dose selection, 35 participants per group) compared CoQ10 doses of 1,800 and 2,700 mg/day. Stage 2 (futility test, 75 patients per group) compared the dose selected in Stage 1 against placebo. In stage 1 , the dose was 2,700 $\mathrm{mg} /$ day. In Stage 2, the pre-specified primary null hypothesis that this dose is superior to placebo was not rejected. It was rejected, however, in an accompanying pre-specified sensitivity test, and further supplementary analyses. Pre-specified secondary analyses showed no significant differences between CoQ10 at 2,700 $\mathrm{mg}$ /day and placebo without any safety concerns. The finding indicated that CoQ10 at 2,700 mg daily for 9 months shows insufficient promise to warrant Phase III testing. Given this outcome, the adaptive Phase II design incorporating a dose selection and a futility test avoided the need for a much larger conventional Phase III trial. The most important weakness in this trial was that no consideration was given for bioavailability and bioactivity of CoQ10 preparation used in the trial. The drug may be highly bioavailable but may not be bioactive which may depend on time of administration and circadian periodicity of neuronal mitochondrial functions. It seems that using highly solubilized ubiquinol could have been more promising, because it is highly bioavailable and can more efficiently incorporate in the neuronal cell membranes of the mitochondria and provide greater benefit. Serum levels of CoQ10 may not be altered in ALS but mitochondrial level may be decreased, hence tissue concentration of CoQ10 appears to be determinant of clinical manifestations of ALS $[27,28]$. It is also possible that sporadic ALS patients may have significantly higher levels of oxidized CoQ10 [28]. Oxidized CoQ10 acts as oxidant and may enhance the generation of superoxide and hydrogen peroxide. Hydrogen peroxide can then react with iron-rich cytochromes to form hydroxyl free radicals which may be damaging to mitochondria of the neurons [28-30]. Therefore, it is important to conduct trials with solubilized ubiquinol in sporadic ALS, before we conclude that CoQ10 trial avoided the need for phase III trial [23].Secondly, ubiquinol may enhance the availability of antioxidant enzymes; superoxide dismutase (SOD), SOD 1 genes and catalase more efficiently compared to commonly used CoQ10. Inclusion of more patients with rapidly progressive ALS toxicity, in the intervention group compared to control group can give false impression that test agent is not better than placebo which is another possible cause of no significant benefit in the above trial [23].

Serum levels of Co Q10 and the Co Q10/cholesterol ratio, were compared in 30 patients with ALS and 42 matched controls using a high performance liquid chromatography technique [27]. The mean serum Co Q10 levels and the Co Q10/cholesterol ratio did not differ significantly between the 2 study groups. Clinical form (spinal vs. bulbar) of ALS, showed no effect on these values and they did not correlate with age, age at onset, and duration of the disease. It is possible that serum Co Q10 concentrations are unrelated with the risk for ALS. Plasma redox status of Co Q10 were compared in 20 sporadic ALS patients with those in 20 healthy age/sex-matched controls [28]. A significant increase in the oxidized form of Co Q10
(sALS=109.3+/-95.2 nM; controls=23.3+/-7.5 nM, $\mathrm{P}=0.0002)$ and in the ratio of oxidized form of Co Q10 to total Co Q10 (\%CoQ10) (sALS $=12.0+/-9.3 \%$; controls $=3.2+/-0.9 \%, \quad \mathrm{P}<0.0001)$ were observed. The \%CoQ10 correlated significantly with the duration of illness (rho=0.494, $\mathrm{P}=0.0315$ ) indicating systemic oxidative stress in the pathogenesis of sALS. It seems that mitochondrial oxidative damage contributes to the pathogenesis of sporadic ALS (sALS) [29]. High-performance liquid chromatography with an electrochemical detector is used to measure the concentrations of the reduced and oxidized forms of CoQ10 in the cerebrospinal fluid (CSF) [29, 30]. There were 30 patients with sALS and 17 age-matched controls with no neurological diseases. The percentage of oxidized CoQ10 in the CSF of sALS patients were significantly greater than those in the CSF of controls $(\mathrm{P}<0.002)$ and were negatively correlated with duration of illness (rho $=-0.64, \mathrm{P}<0.001$ ). These results suggest that mitochondrial oxidative damage contributes to the pathogenesis of sporadic ALS. In Alzheimer's disease (AD), mitochondrial oxidative damage, oxidative DNA damage or both contribute to the neurodegenerative process [30]. A similar method was used to measure the concentrations of the reduced and oxidized forms of CoQ10 and 8-hydroxy-2'-deoxyguanosine (8OHdG) in the cerebrospinal fluid (CSF) of 30 patients with $\mathrm{AD}$ and in 30 age-matched controls with no neurological disease. The percentage of oxidized/total CoQ10 (\%CoQ10) in the CSF of the AD group (78.2 +/- 18.8\%) was significantly higher than in the control group $(41.3+/-$ $10.4 \%)(\mathrm{P}<0.0001)$. The concentration of 8-OHdG in the CSF of AD patients was greater than in the CSF of controls $(\mathrm{P}<0.0001)$ and was positively correlated with the duration of illness $(\mathrm{r}(\mathrm{s})=0.95, \mathrm{P}<0.0001)$. The $\%$ CoQ10 was correlated with concentrations of 8-OHdG in the CSF of AD patients $(\mathrm{r}(\mathrm{s})=0.66, \mathrm{P}<0.001)$. It seems that both mitochondrial oxidative damage and oxidative DNA damage play important roles in the pathogenesis of early $\mathrm{AD}$ development [30, 31]. Recently CoQ10 levels have been reported in CSF among patients without neurological diseases, which may further high light the role of CoQ10 in neuro- degenerative diseases [32].

ALS is a disease causing degeneration of motor neurons in the Betz cells, in the motor areas of the cerebral cortex, brain stem and spinal cord. Most neurodegenerative diseases have defects in the neuronal inner mitochondrial membrane and in oxidative phosphorylation [6-10]. A decrease in CoQ10 levels and in complex I activity have also been reported; leading to the hypothesis that such changes may play a role in the pathogenesis of neuronal damage leading to neuronal degeneration [4-8]. Free radical stress, inflammation and mitochondrial damage as well as abnormality in energy metabolism have been observed in neurodegenerative diseases [6-13]. The neurons in the brain and spinal cord are vulnerable to free radical attack, because they are highly oxygenated structures and contain large amount of iron and polyunsaturated fatty acids and poor antioxidant systems [3-8]. Deficiency of antioxidants, particularly CoQ10 in the neurons may increase the vulnerability of neuronal membrane mitochondria to damage resulting into degeneration. 
In our case, degeneration may have occurred in the motor neurons of the spinal cord because clinical findings do not indicate mental dysfunction and difficulty in swallowing and talking which appear to be indicators of degeneration of brain and brain stem. It has been suggested that most cases of ALS are sporadic but it may also occur in families due to interaction of genetic and environmental factors [3]. CoQ10 is mainly synthesized in the muscles and therefore it may be poorly synthesized due to muscle wasting (1). A coexisting deficiency of CoQ10, owing to increased utilization to fight the oxidative stress due to disease process, may enhance the progression of muscle wasting. In view of this fact, it is possible to propose that CoQ10 may inhibit the progression of muscle wasting and muscle power in patients with ALS as observed in our case.

CoQ10 administration could be useful in patients with Parkinson,s disease, Friedreich's Ataxia, Huntington,s disease, Alzheimers,s disease and ALS [13-23]. Experimental studies indicate that CoQ10 inhibits oxidative stress in mice and in vitro, and diminish damage to the hippocampus following carotid ligation or toxic injury [24]. Endothelin models of cerebral ischemia in rats showed the neuroprotective effects of CoQ10 on lactate acidosis, ATP production, oxidized and reduced glutathione ratio, and super oxide dismutase activities after induced cerebral ischemia [25]. Ren and colleagues [26], explored the effects of CoQ10 on 16 dogs and the results showed that dogs pretreated with CoQ10 had higher levels of ATP and greater protection of the cortex from structural damage. The experimental group had significantly less free radical formation at 60 minutes after the arrest and at 30 minutes into reperfusion than control. Combined, the results from these studies suggest that CoQ10 shows promise as a potent antioxidant and as a free radical scavenger. Oxygen-derived free radicals and abnormal energy metabolism play a role in brain ischemia/reperfusion injury and that CoQ10 exerted a neuron-protective effect by improving brain energy metabolism and diminishing ischemic damage [26]. In a recent study [8] CoQ10 was given to transgenic mice with the P301S tau mutation, which causes fronto-temporal dementia in man. These mice develop tau hyperphosphorylation and neurofibrillary tangles in the brain. Treatment with CoQ10 improved survival and behavioral deficits in the P301S mice. There was a modest reduction in phosphorylated tau in the cortex of P301S mice. The effects of Co Q10 treatment on the electron transport chain enzymes, the mitochondrial antioxidant enzymes, and the tricarboxylic acid cycle were also examined. There was a significant increase in complex I activity and protein levels, and a reduction in lipid peroxidation. The data showed that Co Q10 significantly improved behavioral deficits and survival in transgenic mice with the P301S tau mutation, upregulated key enzymes of the electron transport chain, and reduced oxidative stress.

In a experimental study, feeding with Co Q10 increased cerebral cortex concentrations in 12- and 24-month-old rats [6]. In 12-month-old rats, administration of Co Q10 resulted in significant increases in cerebral cortex mitochondrial concentrations of Co Q10. Oral administration of Co Q10 markedly attenuated striatal lesions produced by systemic administration of 3-nitropropionic acid and significantly increased life span in a transgenic mouse model of familial ALS. These results show that oral administration of Co Q10 increases both brain and brain mitochondrial concentrations. They provide further evidence that Co Q10 can exert neuroprotective effects that might be useful in the treatment of neurodegenerative diseases because CoQ10 is a critical component of the electron transport chain of mitochondria which is main target in causing neurodegeneration [30].

SOD 1 is an enzyme that plays a role in detoxifying free radicals. The pathophysiological mechanisms of both familial and sporadic ALS are unknown, although growing evidence suggests that skeletal muscle tissue is a primary target of ALS toxicity [33]. Skeletal muscle biopsies were performed on transgenic SOD1(G93A) mice, a mouse model of ALS, to determine genetic biomarkers of disease longevity. Consistent with the results obtained from total skeletal muscle of transgenic SOD1(G93A) mice and 74day-old denervated mice, five genes (Mef2c, Gsr, Col19a1, Calm1 and Snx10) could be considered potential genetic biomarkers of longevity in transgenic SOD1(G93A) mice. These results may lead to the exploration of previously unexamined tissues in the search for new disease biomarkers and even to the application of these findings in human studies. It seems that ubiquinol might have greater beneficial effects on genetic expressions that control neuronal degeneration in ALS. In a few familiar forms of ALS, mutations in the SOD1 gene have been held responsible for the degeneration of motor neurons [34]. Nevertheless, after the discovery of the SOD1 mutations no consensus has emerged as to which cells, tissues and pathways are primarily implicated in the pathogenic events that lead to ALS. The recent discovery of mutations in a pair of DNA/RNA-binding proteins called TDP-43 and FUS/TLS as causes of familial and sporadic forms of ALS may be important. Ubiquitous overexpression of mutant SOD1 in transgenic animals recapitulates the pathological features of ALS. It seems that several fundamental issues are still controversial; are the motor neurons the first direct targets of ALS; and what is the contribution of non-neuronal cells, if any, to the pathogenesis of ALS? These studies indicate that CoQ10 appears to be a potential neuroprotectant and may protect against ALS toxicity and enhance survival. Our patient (TK) also has a long survival of 12 years after the diagnosis of ALS in the year 2000 which indicate that phase III trials should be done to find out the effects of ubiquinol on survival. ALS is a fatal disease, with median survival of 3-5 years and cause of deaths are; rapidly progressive ALS, aspiration pneumonia and medical complications of immobility which contribute to morbidity in patients with ALS [35-38]. About 15\% of patients with ALS live 5 years after diagnosis, and about 5\% survive for more than 10 years. Long-term survival is associated with a younger age at onset, being male, and limb rather than bulbar symptom onset and better muscle mass on ultrasonography [38]. Inclusion of more rapidly progressive ALS patients in the intervention group can also resemble no benefit by the 
intervention agent in the experimental group compared to placebo group [23, 39]. CoQ10 may also protect against wasting in ALS because it is rich in muscles and also protect against cardiovascular diseases $[1,40]$. In brief, it is possible that ubiquinol CoQ10 can provide beneficial effects in patients with ALS and hence further trials are necessary using highly solubilized ubiquinol in the treatment.

\section{ACKNOWLEDGEMENTS}

Acknowledgements are due to Mr Raj Chopra, Tishcon Corporation, Westbury, NY, for providing solubilized ubiquinol for treatment.

\section{CONFLICT OF INTEREST}

The authors confirm that this article content has no conflicts of interest.

\section{REFERENCES}

[1] Fedacko J, Pella D, Fedackova E, et al. Coenzyme Q10 in Heart and Brain Diseases. Open Nutra J 2011; 4: 69-87.

[2] Singh RB, Niaz MA, Ghosh S, et al. Dietary intake and plasma levels of antioxidant vitamins in health and disease; a hospital based case control study. J Nutr Environ Med 1995; 5: 235-42.

[3] Singh RB, Singh MM. Effects of coenzyme Q10 in new indications with antioxidant vitamins deficiency. J Nutr Environ Med 1999; 9: 223-8.

[4] Young AJ, Johnson S, Steffens DC, Doraiswami PM. Coenzyme Q10; a review of its promise as neuroprotectant. CNS Spectr 2007; 12: 62-8.

[5] Mancuso M, Orsucci D, Volpi L, Calsolaro V, Siciliano G. Coenzyme Q10 in neuromuscular and neurodegenerative disorders. Curr Drug Targets 2010; 11: 111-21.

[6] Matthews RT, Yang L, Browne S, Baik M, Beal MF. Coenzyme Q10 administration increases brain mitochondrial concentrations and exerts neuroprotective effects. Proc Natl Acad Sci U S A 1998; 95(15): 8892-7.

[7] Beal MF. Bioenergetic approaches for neuroprotection in Parkinson's disease. Ann Neurol 2003; 53(Suppl 3): S39-47.

[8] Elipenahli C, Stack C, Jainuddin S, et al. Behavioral improvement after chronic administration of coenzyme Q10 in P301S transgenic mice. J Alzheimers Dis 2012; 28(1): 173-82.

[9] Shults CW, Oakes D, Kieburtz K, et al. Effects of coenzyme Q10 in early Parkinson disease: evidence of slowing of the functional decline. Arch Neurol 2002; 59: 1541-50.

[10] Sano M, Ernesto C, Thomas RG, et al. A controlled trial of selegiline, alpha-tocopherol, or both as treatment for Alzheimer's disease. The Alzheimer's Disease Cooperative Study. N Engl J Med 1997; 336: 1216-22.

[11] Ruehl WW, Bruyette DS, DePaoli A, et al. Canine cognitive dysfunction as a model for human age-related cognitive decline, dementia and Alzheimer's disease: clinical presentation, cognitive testing, pathology and response to 1-deprenyl therapy. Prog Brain Res 1995; 106: 217-25.

[12] Delanty N, Dichter MA. Oxidative injury in the nervous system. Acta Neurol Scand 1998; 98: 145-53.

[13] Walker FO, Raymond LA. Targeting energy metabolism in Huntington's disease. Lancet 2004; 364: 312-3.

[14] Ebadi M, Govitrapong P, Sharma S, et al. Ubiquinone (coenzyme q10) and mitochondria in oxidative stress of Parkinson's disease. Biol Signals Recept 2001; 10: 224-53.

[15] Shults CW. Coenzyme Q10 in neurodegenerative diseases. Curr Med Chem 2003; 10: 1971-2.

[16] Cooper JM, Schapira AH. Friedreich's Ataxia: disease mechanisms, antioxidant and Coenzyme Q10 therapy. Biofactors 2003; 18: $163-71$.
[17] Feigin A, Kieburtz K, Como P, et al. Assessment of coenzyme Q10 tolerability in Huntington's disease. Mov Disord 1996; 11: 321-3.

[18] Shults CW, Haas RH, Passov D, Beal MF. Coenzyme Q10 levels correlate with the activities of complexes I and II/III in mitochondria from parkinsonian and nonparkinsonian subjects. Ann Neurol 1997; 42: 261-4.

[19] Shults CW, Flint Beal M, Song D, Fontaine D. Pilot trial of high dosages of coenzyme Q10 in patients with Parkinson's disease. Exp Neurol 2004; 188: 491-4.

[20] Muller T, Buttner T, Gholipour AF, Kuhn W. Coenzyme Q10 supplementation provides mild symptomatic benefit in patients with Parkinson's disease. Neurosci Lett 2003; 8: 201-4.

[21] Strijks E, Kremer HP, Horstink MW. Q10 therapy in patients with idiopathic Parkinson's disease. Mol Aspects Med 1997; 18(Suppl): S237-40.

[22] Ferrante KL, Shefner J, Zhang $\mathrm{H}$, et al. Tolerance of high-dose (3,000 mg/day) coenzyme Q10 in ALS. Neurology 2005; 65: 18346.

[23] Kaufmann P, Thompson JLP, Levy G, et al. Phase II trial of CoQ10 for ALS finds insufficient evidence to justify Phase III. Ann Neurol 2009; 66: 235-44.

[24] Piotrowski P, Wierzbicka K, Smialek M. Neuronal death in the rat hippocampus in experimental diabetes and cerebral ischaemia treated with antioxidants. Folia Neuropathol 2001; 39: 147-54.

[25] Ostrowski R, Piotrowski P, Pankowska T, Smialek M. Evaluation of morphological changes after treatment with coenzyme Q10 (CoQ10) in endothelin-1 induced experimental ischemia in the rat. Folia Neuropathol 1998; 36: 185-8.

[26] Ren Z, Ding W, Su Z, et al. Mechanisms of brain injury with deep hypothermic circulatory arrest and protective effects of coenzyme Q10. J Thorac Cardiovasc Surg 1994; 108: 126-33.

[27] Molina JA, de Bustos F, Jiménez-Jiménez FJ, et al. Serum levels of coenzyme Q10 in patients with amyotrophic lateral sclerosis. J Neural Transm 2000; 107(8-9): 1021-6.

[28] Sohmiya M, Tanaka M, Suzuki Y, Tanino Y, Okamoto K, Yamamoto Y. An increase of oxidized coenzyme Q-10 occurs in the plasma of sporadic ALS patients. J Neurol Sci 2005; 228(1): 49-53.

[29] Murata T, Ohtsuka C, Terayama Y. Increased mitochondrial oxidative damage in patients with sporadic amyotrophic lateral sclerosis. J Neurol Sci 2008; 267: 66-9.

[30] Isobe C, Abe T, Terayama Y. Levels of reduced and oxidized coenzyme Q-10 and 8-hydroxy-2'-deoxyguanosine in the CSF of patients with Alzheimer's disease demonstrate that mitochondrial oxidative damage and/or oxidative DNA damage contributes to the neurodegenerative process. J Neurol 2010; 257: 399-404.

[31] Galpern WR, Cudkowicz ME. Coenzyme Q treatment of neurodegenerative diseases of aging. Mitochondrion 2007; 7(suppl): S14653.

[32] Singh RB, Naini A. Cerebrospinal Fluid (CSF) Concentration of coenzyme q10 in clinical practice, a breaking news finding in humans. Open Nutra J 2009; 2: 13-15.

[33] Calvo AC, Manzano R, Atencia-Cibreiro G, et al. Genetic biomarkers for ALS disease in transgenic SOD1(G93A) mice. PLoS One 2012; 7(3): e32632.

[34] Musaro A. State of the art and the dark side of amyotrophic lateral sclerosis. World J Biol Chem 2010; 1: 62-8.

[35] Zaldivar T, Gutierrez J, Lara G, Carbonara M, Logroscino G, Hardiman O. Reduced frequency of ALS in an ethnically mixed population: a population-based mortality study. Neurology 2009; 72(19): 1640-5.

[36] Kimura F, Fujimura C, Ishida S, et al. Progression rate of ALSFRS-R at time of diagnosis predicts survival time in ALS Neurology 2006; 66(2): 265-7.

[37] Zoccolella S, Beghi E, Palagano G, et al. Analysis of survival and prognostic factors in amyotrophic lateral sclerosis: a population based study. J Neurol Neurosurg Psychiatry 2008; 79: 33-7.

[38] Arts IMP, Overeem S, Pillen S, Schelhaas HJ, Zwarts MJ. Ultrasonography to predict survival in amyotrophic lateral sclerosis. J Neuro Neurosurg Psychiatry 2010; 82: 552. 
[39] de Carvalho M, Swash M. Can selection of rapidly progressing patients shorten clinical trials in amyotrophic lateral sclerosis? Arch Neurol 2006; 63: 557-60.
[40] Fedacko J, Moshiri M, Singh RB. Coenzyme Q10 in hypertension. World Heart J 2010; 2: 313-8.

Received: September 17, 2012

Revised: September 22, 2012

Accepted: September 24, 2012

(c) Kawasaki et al.; Licensee Bentham Open.

This is an open access article licensed under the terms of the Creative Commons Attribution Non-Commercial License (http://creativecommons.org/licenses/by-nc/3.0/) which permits unrestricted, non-commercial use, distribution and reproduction in any medium, provided the work is properly cited. 